\title{
HD 19993 and HD 29035: New bright A-type emission-line stars
}

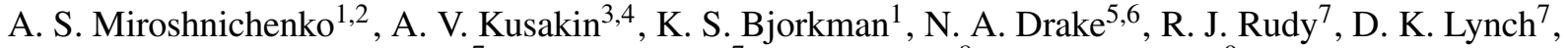 \\ S. Mazuk ${ }^{7}$, C. C. Venturini ${ }^{7}$, R. C. Puetter ${ }^{8}$, and R. B. Perry ${ }^{9}$ \\ ${ }^{1}$ Ritter Observatory, Dept. of Physics \& Astronomy, The University of Toledo, Toledo, OH 43606-3390, USA \\ 2 Pulkovo Observatory, Saint-Petersburg, 196140, Russia \\ 3 Sternberg Astronomical Institute, Universitetskij pr. 13, Moscow, 119899, Russia \\ ${ }^{4}$ Fesenkov Astrophysical Institute, Almaty, 480068, Kazakhstan \\ 5 Observatório Nacional/MCT, Rua General José Cristino 77, São Cristovão, Rio de Janeiro, 20921-400, Brazil \\ 6 Sobolev Astronomical Institute, St. Petersburg University, Universitetsky Pr. 28, Petrodvorets, St. Petersburg, 198504, Russia \\ 7 The Aerospace Corp. M2/266, PO Box 92957, Los Angeles, CA 90009, USA \\ ${ }^{8}$ Center for Astrophysics and Space Science, University of California, San Diego, C-0111, La Jolla, \\ CA 92093, USA \\ 9 Earth and Space Science Support Office, M/S 160, NASA Langley Research Center, Hampton, VA 23681, USA
}

Received 10 June 2003 / Accepted 25 August 2003

\begin{abstract}
We present the results of photometric and spectroscopic observations of two A-type stars (HD 19993 and HD 29035), optical counterparts of the IRAS sources (F03108+3729 and 04331+5211, respectively). H $\alpha$ line emission is found in the optical spectra of both objects for the first time. Our analysis of the spectral line profiles and the photometric information shows that HD 19993 is an A7/8 II $\operatorname{star}\left(T_{\text {eff }} \sim 7500 \pm 200 \mathrm{~K}, \log g=2.7 \pm 0.2, v \sin i=35 \pm 2 \mathrm{~km} \mathrm{~s}^{-1}, M=4.8 \pm 0.8 M_{\odot}\right)$ with a mild metal deficit $([\mathrm{Fe} / \mathrm{H}] \sim-0.3 \pm 0.1)$, while HD 29035 is a B9/A0 V star $\left(T_{\text {eff }} \sim 10000 \mathrm{~K}, \log g \sim 4, v \sin i \sim 150 \mathrm{~km} \mathrm{~s}^{-1}\right)$. The presence of an IR excess and the $\mathrm{H} \alpha$ emission in combination with a very different location in the Hertzsprung-Russell diagram suggest the different nature and evolutionary stages of the objects. We conclude that HD 29035 is a candidate Herbig Ae type star, a possible member of a group of similar objects. HD 19993 seems to be a post-main-sequence object.
\end{abstract}

Key words. techniques: spectroscopic - techniques: photometric - stars: circumstellar matter stars: individual: HD 19993, HD 29035

\section{Introduction}

The presence of emission lines in the spectra of hot stars indicates that they are surrounded by significant amounts of circumstellar (CS) matter. CS matter also produces continuum emission that lies predominantly in the infrared (IR) region. Both phenomena are usually observed at certain evolutionary stages. From parameters of the line emission such as the intensity, profile shape, and content of observed species, and from the shape and spectral features of the IR excess, it is frequently possible to recognize the evolutionary state of an object. For example, pre-main-sequence Herbig Ae/Be stars display strong near- and far-IR excesses due to CS dust with temperatures $\left(T_{\mathrm{d}}\right)$ less than $\sim 1500 \mathrm{~K}$ in addition to strong emission lines of neutral hydrogen. In contrast, their descendants, Vega-type stars that have already reached the main-sequence, do not show emission-line spectra and only exhibit far-IR excesses due to much colder dust $\left(T_{\mathrm{d}} \leq 100 \mathrm{~K}\right)$. It is important to investigate such stars to refine our knowledge of stellar evolution and of

Send offprint requests to: A. S. Miroshnichenko, e-mail: anatoly@physics.utoledo.edu the mechanisms of CS envelope formation. There are still few hot stars with well-studied CS environments, and discoveries of new objects bring us closer to these goals.

Several thousand early-type emission-line stars were discovered during low-dispersion surveys conducted during the first half of the twentieth century (such as the Mount Wilson survey, Merrill \& Burwell 1933). Current catalogs contain more than 7000 such stars (Reed 1998), most of which are brighter than $V \sim 13 \mathrm{mag}$. With the advent of large telescopes and very sensitive detectors, it is much easier to detect a new faint emission-line star rather than a bright one (with $V \leq$ $10 \mathrm{mag}$ ), because most of the latter have already been discovered. However, since line emission can be variable, such discoveries can occur (e.g., Coté \& van Kerkwijk 1993).

A fruitful approach in searching for new emission-line stars is to take optical spectra of stars with IR excesses. The IRAS satellite detected nearly a quarter of a million point sources in the Milky Way, some of which were identitified with previously unknown hot emission-line stars (e.g., Oudmaijer et al. 1992). Our observations of some objects from the list of Oudmaijer et al. resulted in the discovery of 2 bright Be stars 
Table 1. Initial information and IRAS data for HD 19993 and HD 29035.

\begin{tabular}{|c|c|c|c|c|c|c|c|c|c|c|}
\hline HD & Sp.T. & $\begin{array}{c}V_{\mathrm{T}} \\
\mathrm{mag}\end{array}$ & $\begin{array}{l}(B-V)_{\mathrm{T}} \\
\text { mag }\end{array}$ & IRAS & $\begin{array}{c}F_{12} \\
\text { Jy }\end{array}$ & $\begin{array}{c}F_{25} \\
\text { Jy }\end{array}$ & $\begin{array}{c}F_{60} \\
\text { Jy }\end{array}$ & $\begin{array}{c}F_{100} \\
\text { Jy }\end{array}$ & $\begin{array}{c}\text { Offset } \\
\prime \prime\end{array}$ & $\begin{array}{c}\text { Excess } \\
\text { mag }\end{array}$ \\
\hline 1 & 2 & 3 & 4 & 5 & 6 & 7 & 8 & 9 & 10 & 11 \\
\hline 19993 & $\mathrm{~A} 2$ & 8.32 & 0.56 & F03108+3729 & $0.43 \pm 0.02$ & $0.22 \pm 0.04$ & $0.11 \pm 0.07$ & & 5.3 & 1.31 \\
\hline 29035 & A0 & 8.81 & 0.29 & $04331+5211$ & $0.78 \pm 0.03$ & $1.05 \pm 0.03$ & $5.05 \pm 0.08$ & $9.6 \pm 0.7$ & 22.3 & 2.26 \\
\hline
\end{tabular}

The star IDs and spectral types according to the HD catalog are listed in Cols. 1, 2, the Tycho-2 (Hog et al. 2000) $B V$ photometry in Cols. 3 , 4, the IRAS ID according to the Faint (F) or Point Source Catalog in Col. 5, the IRAS fluxes measured using the ADDSCAN procedure in Cols. 6-9, the offset between the optical and IRAS positions in Col. 10, and the colour-excess $E(V-[12])$ in Col. 11.

Table 2. Multicolour photometry of HD 19993 and HD 29035.

\begin{tabular}{cccccccccc}
\hline \hline HD & $V$ & $W-B$ & $U-B$ & $B-V$ & $V-R$ & $J$ & $H$ & $K$ & Rem. \\
\hline 19993 & & & & & & $7.00 \pm 0.02$ & 6.80 & 6.70 \\
19993 & $8.26 \pm 0.01$ & $+0.37 \pm 0.02$ & $+0.29 \pm 0.01$ & $+0.50 \pm 0.01$ & $+0.53 \pm 0.01$ & $7.14 \pm 0.15$ & $6.93 \pm 0.15$ & $6.84 \pm 0.15$ & $b$ \\
29035 & & & & & & & \\
29035 & $8.78 \pm 0.01$ & $+0.09 \pm 0.02$ & $+0.07 \pm 0.01$ & $+0.27 \pm 0.01$ & $+0.28 \pm 0.01$ & & $7.93 \pm 0.03$ & $7.88 \pm 0.02$ & $c$ \\
\end{tabular}

The $U-B$ colour-indices are calculated from the $W-B$ using a relationship described in the text. The measurement uncertainties are shown as subscript indices in hundredths of a magnitude. The uncertainties of the $H$ and $K$ magnitudes are not listed in the 2MASS catalog for HD 19993, because of a small number of measurements.

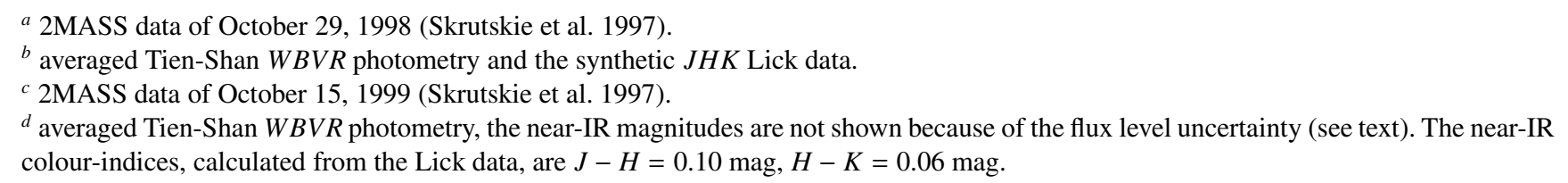

(HD 4881 and HD 5839, Miroshnichenko et al. 1999). In the same paper, we also reported our finding of a new Vega-type star, HD 184761, as a result of our own cross-identification of the Henry Draper (HD) catalog and the IRAS Point Source Catalog. Later we undertook a new cross-identification of HD and the IRAS Point and Faint Source Catalogs and found more than 30 previously unreported B, A, and F-type stars with IR excesses. In this search we followed a procedure outlined by Mannings \& Barlow (1998).

Follow-up spectroscopic observations of most of them showed that only 2 of these objects (HD 19993 and HD 29035) exhibit emission lines. In this paper we report the results of our observations of these two stars, while the non-emission objects (which have a different nature and evolutionary state) will be described elsewhere.

The information that has been published previously about both stars is presented in Table 1 . The infrared excesses $E(V-$ $[12])=\left(V_{0}-[12]_{\mathrm{obs}}\right)-\left(V-[12]_{\text {intr }}\right)\left(\right.$ where $V_{0}$ is the dereddened $V$-band brightness, [12] is the IRAS $12-\mu \mathrm{m}$ band magnitude, and $V-[12]_{\text {intr }}$ is the intrinsic colour-index for the corresponding spectral type from Waters et al. 1987) listed in Col. 11 are significant. We used the ADDSCAN technique (co-addition of all one-dimensional IRAS scans through the object's position, see Weaver \& Jones 1992) to check for extended emission from the objects. The images of HD 19993 are slightly extended with respect to the point source templates at 12 and $25 \mu \mathrm{m}$. At 60 and $100 \mu \mathrm{m}$ it is contaminated by a close extended source, so that only the 60- $\mu$ m flux can be recovered. HD 29035 is extended in all the IRAS bands.
In Sect. 2 we describe our observations of the stars, in Sect. 3 we present an analysis of the object's properties, and in Sect. 4 we discuss their nature and evolutionary state.

\section{Observations}

The differential photometric observations of HD 19993 and HD 29035 were obtained at a $0.5-\mathrm{m}$ telescope at the TienShan Observatory of the Fesenkov Astrophysical Institute in Kazakhstan, using a standard photometer with a photomultiplier FEU-79, between August 2001 and February 2002 (diaphragm $\left.50^{\prime \prime}\right)$. We used a set of filters very close to those of the $W B V R$ Moscow photometric system (Khaliullin et al. 1985). Comparison stars were taken from a catalog of Kornilov et al. (1991). The BVR-bands of the Moscow system are identical to those of the Johnson system, while the $W$-band is slightly narrower than the Johnson $U$-band and does not include atmospheric ozone bands shortward of $\lambda 3200 \AA$. Typical errors of individual observations, including those of transformation from the instrumental to the standard photometric system, do not exceed 0.02 mag. HD 19993 was observed 5 times, while HD 29035 was observed 3 times. No brightness variations beyond the measurement uncertainties (0.01-0.02 mag) were found for both stars. The results are presented in Table 2.

In order to compare the photometric data obtained in the $W B V R$ system with those in the Johnson system and use existing relations for the spectral types versus colour-indices, we calculated the relation between $U-B$ and $W-B$ for early-type stars using the Bright Star Catalogue (Hoffleit \& Warren 1991) 

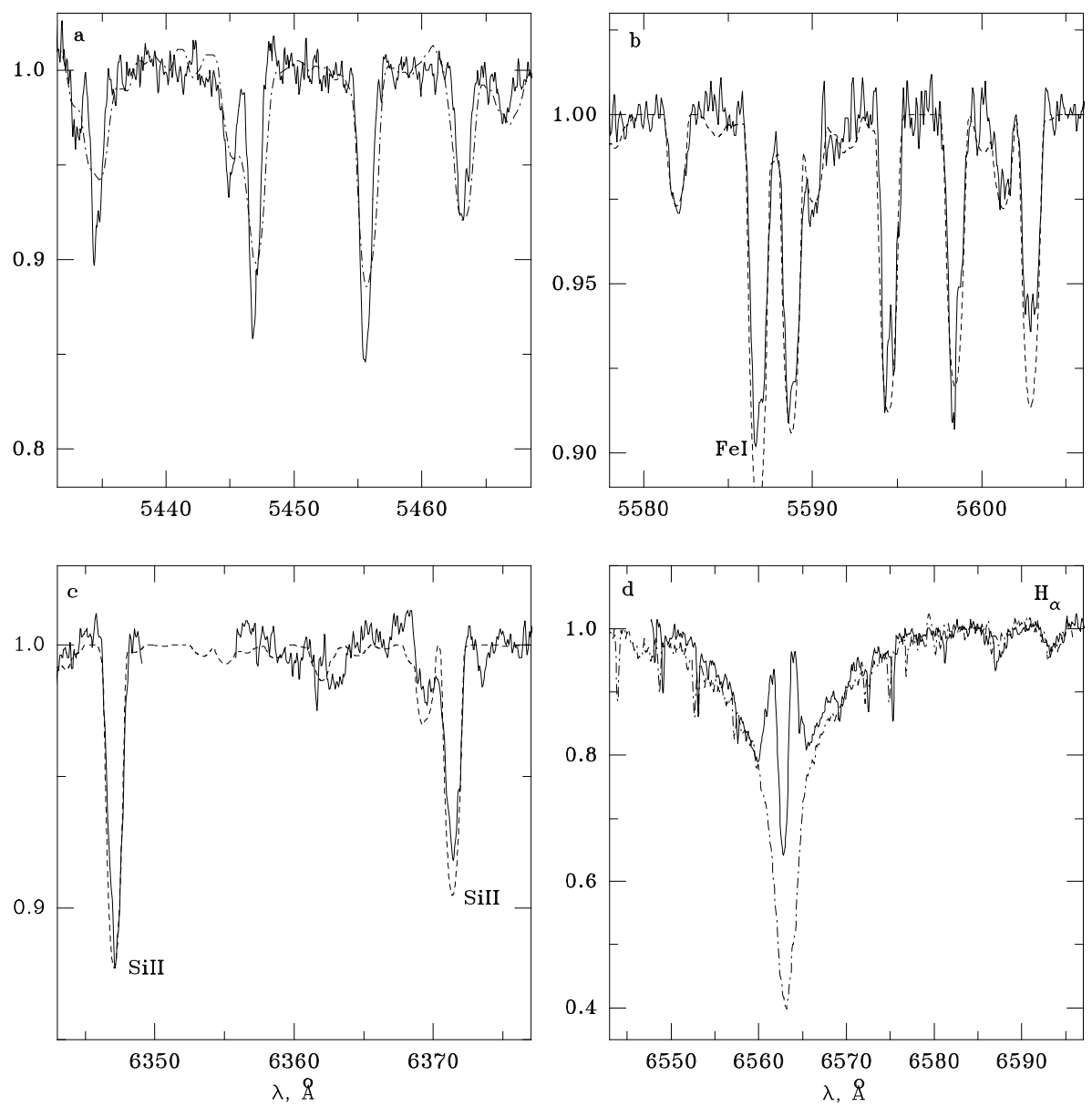

Fig. 1. Portions of the spectrum of HD 19993 (solid line). In panels a) and d) the spectrum of HD 19993 is compared with that of $\rho$ Gem (dot-dashed line), while in panels b) and c) with a theoretical spectrum for $T_{\text {eff }}=7500 \mathrm{~K}, \log g=2.7, v \sin i=35 \mathrm{~km} \mathrm{~s}^{-1}$, and metal abundances from Table 4 (dashed line). All the absorption lines in panel a) are Fe I lines, while the unmarked lines in panel b) are Ca I lines. The intensities are normalized to the underlying continuum, while the wavelengths are given in $\AA$.

and the WBVR catalog by Kornilov et al. (1991). Comparing the two catalogs, we found that for nearly a thousand B7-F2 stars $(U-B)=0.803 \pm 0.004(W-B)-0.005 \pm 0.001$. The $U-B$ colour-indices calculated on the basis of this relationship are shown in Table 2.

The spectroscopic observations were obtained on October 9-12, 2001 and September 23-27, 2002 at the 2.1-m Otto Struve telescope of the McDonald Observatory (Mt. Locke, Texas, USA) with the Sandiford échelle-spectrometer (McCarthy et al. 1993) and a $1200 \times 400$ pixel CCD in the range 5430-6915 $\AA$ with a spectral resolving power $R \sim$ 60000 . The signal-to-noise ratio in continuum exceeds 100 in all the spectra. The data were reduced in IRAF $^{1}$.

High-resolution spectra of several bright stars with known fundamental parameters ( $\rho$ Gem, Vega, $\phi$ Cas) were obtained with a fiber-fed échelle spectrograph and a Wright Instruments Ltd. CCD camera on the 1-m telescope of Ritter Observatory for comparison purposes. The spectra, from 5285 to $6597 \AA$,

${ }^{1}$ IRAF is distributed by the National Optical Astronomy Observatories, which are operated by the Association of Universities for Research in Astronomy, Inc., under contract with the National Science Foundation. consisted of nine non-overlapping $\simeq 70 \AA$ wide orders, with spectral resolving power $R \simeq 26000$.

The infrared low-resolution spectroscopic observations were acquired on December 19, 2002 with the 3-m Shane reflector of the Lick Observatory and the Aerospace nearinfrared imaging spectrograph. The spectrograph, which is described by Rudy et al. (1999), uses two channels to provide wavelength coverage from 0.8 to $2.5 \mu \mathrm{m}$. The blue $(0.8-$ $1.4 \mu \mathrm{m}$, resolution $14 \AA$ ) and red $(1.4-2.5 \mu \mathrm{m}$, resolution $36 \AA$ A) spectra were acquired simultaneously. A more detailed technical description of this type of observation is given by Miroshnichenko et al. (2000). A $2^{\prime \prime}$ slit was employed for the observations of both stars and its calibrator star HR 1489, a G0 dwarf ( $V=5.99 \mathrm{mag})$. The objects and the calibrator were observed at almost the same air mass (1.00 for HR 1489, 1.02 for HD 19993, and 1.04 for HD 29035). The instrumental response and most of the effects of atmospheric absorption were removed by dividing the spectra of the stars by that of the calibrator. To remove the intrinsic spectrum of the calibrator from this ratio, a model from Kurucz (1994) appropriate to a G0 V star was used. The presence of some clouds during the observations make the absolute flux level somewhat uncertain, but does not affect the shape of the spectra. 

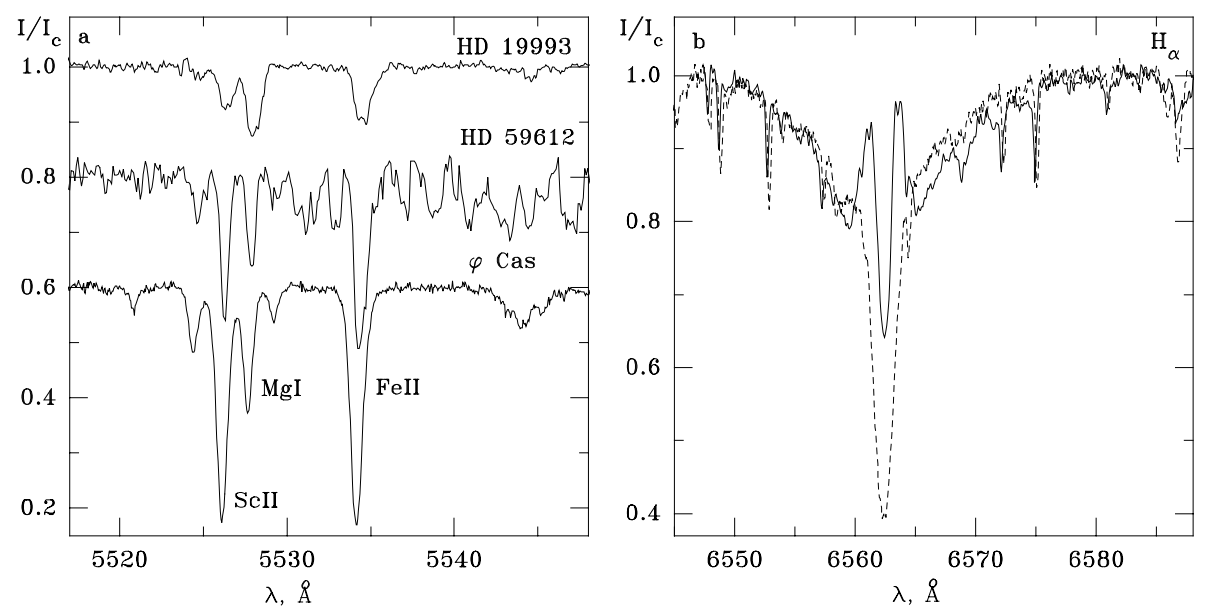

Fig. 2. Comparison of the spectrum of HD 19993 with the spectra of supergiants. In panel b), the H $\alpha$ line profile of HD 19993 is shown by a solid line, while that of $\phi$ Cas is shown by a dashed line. The spectrum of HD 59612 was kindly provided by E. Verdugo. The intensities and wavelengths are given in the same units as in Fig. 1.
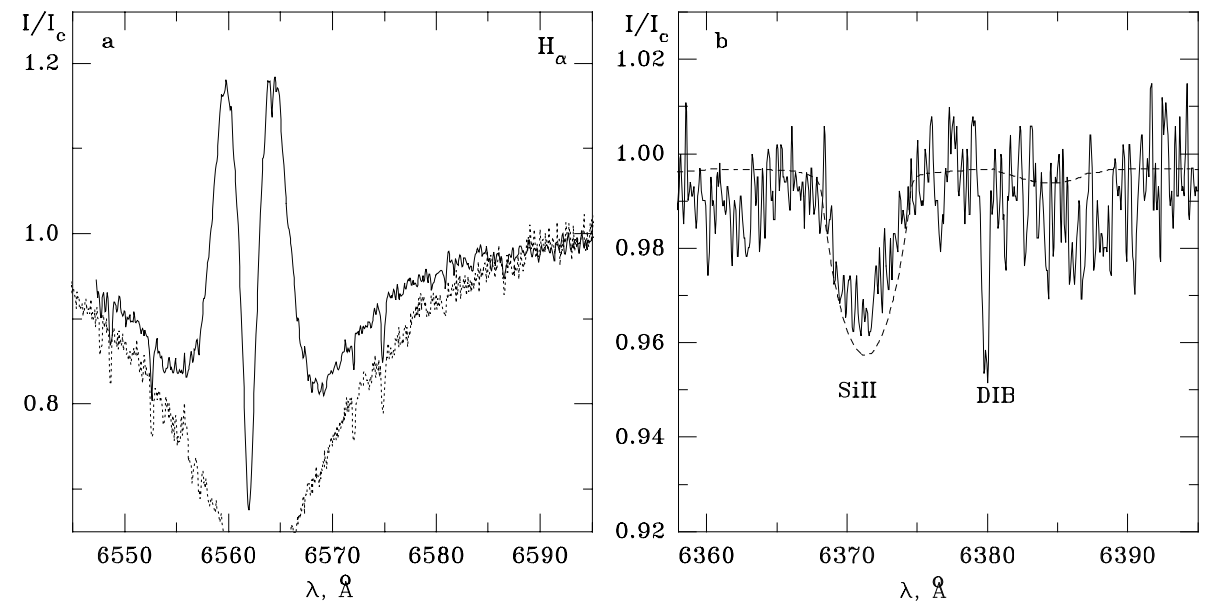

Fig. 3. Portions of the spectrum of HD 29035 (solid lines). Panel a) shows the H $\alpha$ line profile in comparison with that of Vega (dotted line). Panel b) shows the Si II $\lambda 6371 \AA ̊$ line in comparison with a theoretical spectrum for $T_{\text {eff }}=10000 \mathrm{~K}, \log g=4.0, v \sin i \sim 150 \mathrm{~km} \mathrm{~s}^{-1}$, calculated using the code SPECTRUM (dashed line). The intensities and wavelengths are given in the same units as in Fig. 1.

\section{Results}

Our optical spectroscopic data revealed that both HD 19993 and HD 29035 exhibit double-peaked $\mathrm{H} \alpha$ emission (Figs. 2 and 3 , respectively). No other emission lines were detected in the observed spectral range. Also, no noticeable variations in the line properties or content were detected in our optical spectra, which were taken about a year apart.

The near-IR spectra of both objects show only photospheric lines. Their slopes are in agreement with the photometric data (see Table 2). The absorption-line spectra of the two stars, as well as their basic parameters and spectral energy distributions (SED), turned out to be different. In Figs. 1 and 2, portions of the optical spectrum of HD 19993 are compared with those of different stars. In Fig. 3, sections of the optical spectrum of HD 29035 are shown. Both objects' SEDs and portions of their near-IR spectra are shown in Fig. 4. Below we discuss the objects' properties separately.

\subsection{HD 19993}

HD 19993, which has an A2 spectral type in HD, displays absorption lines of neutral (e.g., Fe I, Ca I, Mg I) and singly ionized (e.g., Fe II, Sc II, Ba II, Si II) metals and weak diffuse interstellar bands (DIBs). The spectral line content and the optical colour-indices are consistent with a spectral type A7-F0 and an interstellar reddening of $E(B-V) \sim 0.3$ mag. The Si II lines at 6347 and $6371 \AA$, which are luminosity-dependent in A-type and early F-type stars (e.g., Rosendhal 1974), are weak in the spectrum of HD 19993 (the equivalent widths are 0.14 and $0.10 \AA$, respectively) and suggest a moderately low luminosity for the star. The spectral line widths indicate a small projectional rotation velocity. The mean heliocentric radial velocity of HD 19993 was measured by averaging positions of about 60 absorption lines. In both of our spectra obtained in 2001 and 2002, it is $+1 \pm 4 \mathrm{~km} \mathrm{~s}^{-1}$.

Photometry of the nearby stars shows that there is almost no reddening in this direction at distances $(D)$ closer than $100 \mathrm{pc}$ to the Sun, while at $D \sim 200 \mathrm{pc}$ it reaches 

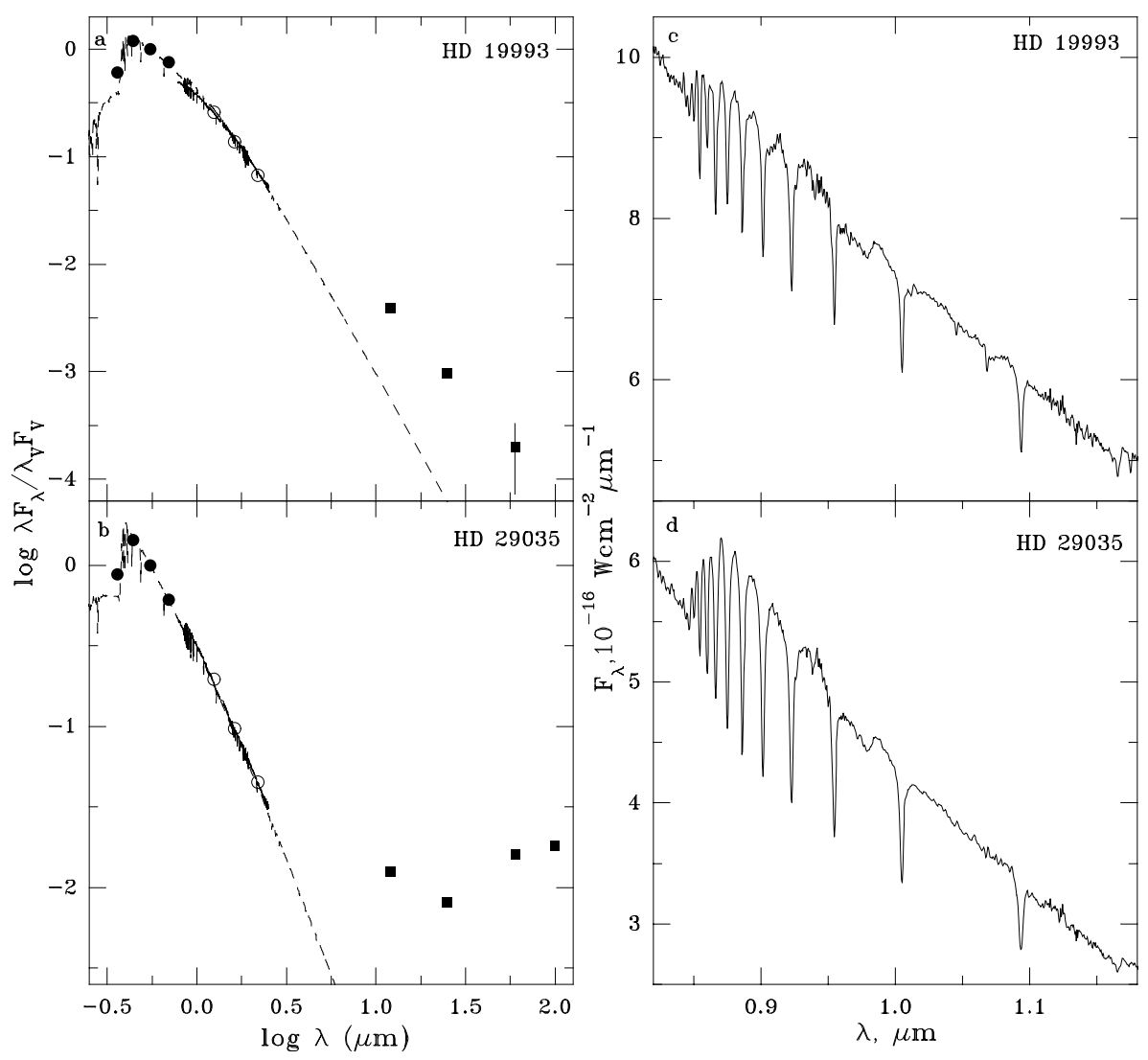

Fig. 4. The spectral energy distributions and portions of the near-IR spectra of HD 19993 and HD 29035. Panels a) and b) show the dereddened SEDs. The optical photometric data are shown by filled circles, 2MASS data by open circles, the IRAS data by filled squares, and near-IR spectra by solid lines. The $E(B-V)=0.35 \mathrm{mag}$ is used for HD 19993 and 0.32 mag for HD 29035. An interstellar extinction law from Savage \& Mathis (1979) was applied to the original data. The near-IR flux of HD 29035 is rescaled to the level of the 2MASS photometry. The theoretical SEDs from Kurucz (1994) are shown by dashed lines. $T_{\text {eff }}=7500 \mathrm{~K}$ and $\log g=2.5$ are adopted for HD 19993 , while $T_{\text {eff }}=10000 \mathrm{~K}$ and $\log g=4.0$ for HD 29035. The fluxes are normalized to that in the $V$-band at $0.55 \mu \mathrm{m}$. Panels $\mathbf{c}$ ) and d) show the blue portion of the near-IR spectra of the objects with hydrogen absorption lines. The fluxes are given in units of $10^{-16} \mathrm{~W} \mathrm{~cm}^{-2} \mu \mathrm{m}^{-1}$ and the wavelengths are given in microns.

$E(B-V) \sim 0.05-0.10 \mathrm{mag}$. The star was observed by HIPPARCOS (ESA 1997), which did not detect any photometric variability $\left(H_{\mathrm{p}}=8.37 \pm 0.02 \mathrm{mag}\right)$ and gave an insignificant parallax $(0.3 \pm 1.0$ mas $)$.

A comparison of our spectrum of HD 19993 with those of late A and early F-type stars shows that $\rho$ Gem, an F0 V star with an effective temperature $T_{\text {eff }}=6970 \pm 70 \mathrm{~K}$ (Barklem et al. 2002), a surface gravity $\log g=4.0$, and a metallicity $[\mathrm{Fe} / \mathrm{H}]=-0.31$ (Alonso et al. 1996), has similar line strengths and content (Fig. 1). The spectral lines of $\rho$ Gem are slightly broader and shallower than those of HD 19993 due to a larger rotational velocity of the former $\left(v \sin i=63 \mathrm{~km} \mathrm{~s}^{-1}\right.$, Uesugi \& Fukuda 1970). The wings of the $\mathrm{H} \alpha$ lines of the two stars are very similar (Fig. 1d). We should note here that due to a curvy instrumental continuum of the échelle orders, the wings' slope of broad lines may be derived with some uncertainties (see also Miroshnichenko et al. 2001).

Nevertheless, with an intrinsic colour-index of an F0 V star ( $B-V=0.36 \mathrm{mag}$, Bessell et al. 1998) and an average luminosity of $\log L / L_{\odot}=0.8$ (Straizhys \& Kuriliene 1981), HD 19993 would have a distance $D=105 \mathrm{pc}$ from the Sun. HIPPARCOS would measure the object's parallax with a good precision at such a close distance in the absence of confusing factors, such as hidden binarity. Therefore, other solutions for the main parameters of HD 19993 need to be considered.

The numerous metal absorption lines suggest $T_{\text {eff }} \leq$ $8000 \mathrm{~K}$. The main luminosity criteria in the observed spectral region are the $\mathrm{Si}$ II lines strength and the $\mathrm{H} \alpha$ wings slope. The former are only consistent with a surface gravity $\log g \geq 2$, while the latter favors $\log g \sim 2-3$ only if $T_{\text {eff }} \geq 7000 \mathrm{~K}$. Figure 2 shows the spectral parts of HD 19993 in comparison with supergiants of different temperatures and luminosities ( $\phi$ Cas, F0 Ia, $T_{\text {eff }}=7200 \mathrm{~K}, \log g=0.4$, Rozenzweig \& Anderson 1993; HD 59612, A6 Ia/II, $T_{\text {eff }}=8500 \mathrm{~K}, \log g=$ 1.5, Verdugo et al. 1999). The supergiants generally exhibit stronger lines of ionized metals and narrower $\mathrm{H} \alpha$ profiles (at $\left.T_{\text {eff }} \leq 8000 \mathrm{~K}\right)$.

To determine the atmospheric parameters of HD 19993, we measured equivalent widths $\left(W_{\lambda}\right)$ of all available neutral and ionized iron lines. After eliminating suspected blends, we used $22 \mathrm{Fe} \mathrm{I}$ and $5 \mathrm{Fe}$ II lines. The oscillator strengths for them were taken from Lambert et al. (1996) if available, otherwise from the NIST database ${ }^{2}$. The iron abundances, as well as synthetic spectra, were calculated using the latest version

\footnotetext{
${ }^{2}$ http://physics.nist.gov/cgi-bin/AtData/main_asd
} 
(April 2002) of the program MOOG (Sneden 1973). Required input model atmospheres were interpolated from the Kurucz (1993). Following an iterative procedure, we derived a microturbulent velocity $\left(\xi_{\mathrm{t}}\right)$ and $T_{\text {eff }}$ by requiring that the abundances calculated for the $\mathrm{Fe} I$ lines do not show any dependence upon $W_{\lambda} / \lambda$ and excitation potential. The derived iron abundance was compared to that of the model atmosphere. If the difference exceeded 0.05 dex, another model was computed via interpolation, and the process was repeated until a consistent result was achieved. Figure 5 shows the iron abundance versus $\log \left(W_{\lambda} / \lambda\right)$ and the lower excitation potential for the $\mathrm{Fe} I$ lines, calculated with the adopted atmospheric model. The correct $\xi_{\mathrm{t}}$ and $T_{\text {eff }}$ are indicated by a zero slope in the plots.

The surface gravity, $\log g$, was derived from the ionization equilibrium of the Fe I and Fe II lines. As a result (after many iterations), we determined the following atmospheric parameters for HD 19993: $T_{\mathrm{eff}}=7500 \pm 200 \mathrm{~K}, \log g=2.7 \pm 0.2, \xi_{\mathrm{t}}=$ $2.9 \pm 0.2 \mathrm{~km} \mathrm{~s}^{-1}$, and $[\mathrm{Fe} / \mathrm{H}]=-0.32 \pm 0.14^{3}$. The solar abundances, $\log \varepsilon(\mathrm{X})_{\odot}$, were taken from Anders \& Grevesse (1989) except for the iron abundance. The value of $\log \varepsilon(\mathrm{Fe})_{\odot}=7.52$ was adopted from Sneden et al. (1991). We also derived the star's projected rotation velocity $v \sin i=35 \pm 2 \mathrm{~km} \mathrm{~s}^{-1}$.

Using the adopted atmospheric parameters, we determined abundances for some other chemical elements using two methods. For the unblended lines, the abundances were derived directly from the measured $W_{\lambda}$ s, while the synthetic spectra were calculated in the regions of blended lines. Atomic parameters were taken from the VALD database (Piskunov et al. 1995; Kupka et al. 1999).

For the carbon abundance determination, we conducted spectrum synthesis in the region of the 6010.7, 6013.2, and 6014.8 Å C I lines, taking into account molecular dissociation equilibrium. All the lines show an underabundance of carbon relative to iron, $[\mathrm{C} / \mathrm{Fe}]=-0.53$. Comparison of the computed spectra with the observed one in the region of the carbon lines is shown in Fig. 6a. The oxygen abundance was determined by fitting a synthetic spectrum in the region of the O I triplet at $6156.0-6158.2 \AA$. Comparison of the computed and observed spectra suggests that oxygen is somewhat underabundant relative to iron $([\mathrm{O} / \mathrm{Fe}]=-0.18)$. Figure $6 \mathrm{~b}$ shows a comparison between the observed and synthetic spectra in the region of O I lines. Despite carbon and oxygen lines being noisy, their weakness confirms the underabundance of these elements. We were unable to determine the nitrogen abundance of HD 19993, since the observed spectral region contains only extremely weak lines of this element. Nevertheless, our tests showed that the high temperature of HD 19993 prevents a dependence of the $\mathrm{C}$ and $\mathrm{O}$ abundances on that of $\mathrm{N}$, since almost no molecules survive in its atmosphere.

The sodium abundance was derived using spectral synthesis in the regions of the three $\mathrm{Na} I$ lines: 5682.6, 5688.2, and $6160.7 \AA$ (see Fig. 6c). The sodium excess of $[\mathrm{Na} / \mathrm{Fe}]=$ +0.3 was found. Sodium is of interest for nucleosynthesis, and its abundance was extensively studied in stellar spectra. A sodium excess was detected in atmospheres of AF-type

\footnotetext{
${ }^{3}$ We adopted the usual spectroscopic notation, namely $[\mathrm{X} / \mathrm{H}]=$ $\log \varepsilon(\mathrm{X})_{\text {star }}-\log \varepsilon(\mathrm{X})_{\odot}$ and $\log \varepsilon(\mathrm{X})=\log \left(N_{\mathrm{X}} / N_{\mathrm{H}}\right)+12.0$.
}

Table 3. Individual abundances of the neutral and ionized iron lines.

\begin{tabular}{|c|c|c|c|c|}
\hline$\lambda(\AA)$ & $\chi(\mathrm{eV})$ & $\log g f$ & $W_{\lambda}(\mathrm{m} \AA)$ & {$[\mathrm{Fe} / \mathrm{H}]$} \\
\hline \multicolumn{5}{|l|}{$\mathrm{Fe} \mathrm{I}$} \\
\hline 5434.524 & 1.01 & -2.12 & 100 & -0.37 \\
\hline 5445.042 & 4.39 & +0.04 & 70 & -0.29 \\
\hline 5446.917 & 0.99 & -1.91 & 140 & -0.04 \\
\hline 5463.280 & 4.43 & +0.09 & 90 & -0.06 \\
\hline 5487.746 & 4.32 & -0.65 & 30 & -0.21 \\
\hline 5497.516 & 1.01 & -2.84 & 60 & -0.13 \\
\hline 5554.895 & 4.55 & -0.38 & 30 & -0.31 \\
\hline 5569.618 & 3.42 & -0.49 & 70 & -0.50 \\
\hline 5572.842 & 3.40 & -0.28 & 100 & -0.35 \\
\hline 5586.756 & 3.37 & -0.12 & 110 & -0.39 \\
\hline 5615.644 & 3.33 & +0.05 & 110 & -0.60 \\
\hline 5624.550 & 3.42 & -0.90 & 50 & -0.34 \\
\hline 5705.990 & 4.10 & -0.53 & 40 & -0.33 \\
\hline 5762.992 & 4.21 & -0.41 & 50 & -0.23 \\
\hline 6020.169 & 4.61 & -0.21 & 40 & -0.27 \\
\hline 6024.070 & 4.55 & -0.06 & 50 & -0.32 \\
\hline 6065.482 & 2.61 & -1.53 & 40 & -0.48 \\
\hline 6191.558 & 2.43 & -1.42 & 60 & -0.46 \\
\hline 6230.723 & 2.56 & -1.28 & 60 & -0.50 \\
\hline 6393.601 & 2.43 & -1.43 & 70 & -0.32 \\
\hline 6421.351 & 2.28 & -2.01 & 40 & -0.26 \\
\hline 6430.846 & 2.18 & -2.01 & 40 & -0.34 \\
\hline \multicolumn{5}{|l|}{$\mathrm{Fe}$ II } \\
\hline 5534.834 & 3.25 & -2.77 & 122 & -0.26 \\
\hline 6239.950 & 3.89 & -3.68 & 24 & -0.20 \\
\hline 6369.464 & 2.89 & -4.19 & 30 & -0.32 \\
\hline 6416.921 & 3.89 & -2.68 & 72 & -0.49 \\
\hline 6432.650 & 2.89 & -3.58 & 73 & -0.36 \\
\hline
\end{tabular}

The laboratory wavelength is listed in Col. 1, the lower excitation potential in Col. 2, the oscillator strength in Col. 3, the $W_{\lambda}$ measurement in Col. 4, and the individual abundance in Col. 5.

supergiants (see reviews in Lambert 1996; Takeda \& TakadaHidai 1981; Venn 1995). According to Denisenkov \& Ivanov (1987), sodium is synthesized in the convective core of mainsequence stars in the $\mathrm{NeNa}$ cycle and, as a result of mixing at the first dredge-up, comes up to the stellar surface along with other products of the $\mathrm{CNO}$ cycle.

The calcium abundance was derived from nine weak and medium $\mathrm{Ca}$ I lines, with $W_{\lambda}$ between 30 and $120 \mathrm{~m} \AA$. For 5 lines we used the spectral synthesis method and for the other 4 lines the equivalent width method was used.

We estimated a dependence of the derived element abundances on the uncertainties of the determined stellar parameters by calculating abundances with the following changes in input parameters: $\pm 200 \mathrm{~K}, \pm 0.2 \mathrm{dex}$, and $0.2 \mathrm{~km} \mathrm{~s}^{-1}$ in $T_{\text {eff }}, \log g$, and $\xi_{\mathrm{t}}$, respectively. The resulting abundance variations are not especially sensitive to the input atmospheric parameters, $\Delta \log \varepsilon(\mathrm{X}) \leq 0.15$, excluding the strong Ba II line. The results of the abundance determinations are presented in Table 4 . The abundance uncertainties, $\sigma_{\text {tot }}$, were calculated as a sum of the squares of the various sources of uncertainties, such as uncertainties in the derived atmospheric parameters and line-to-line 

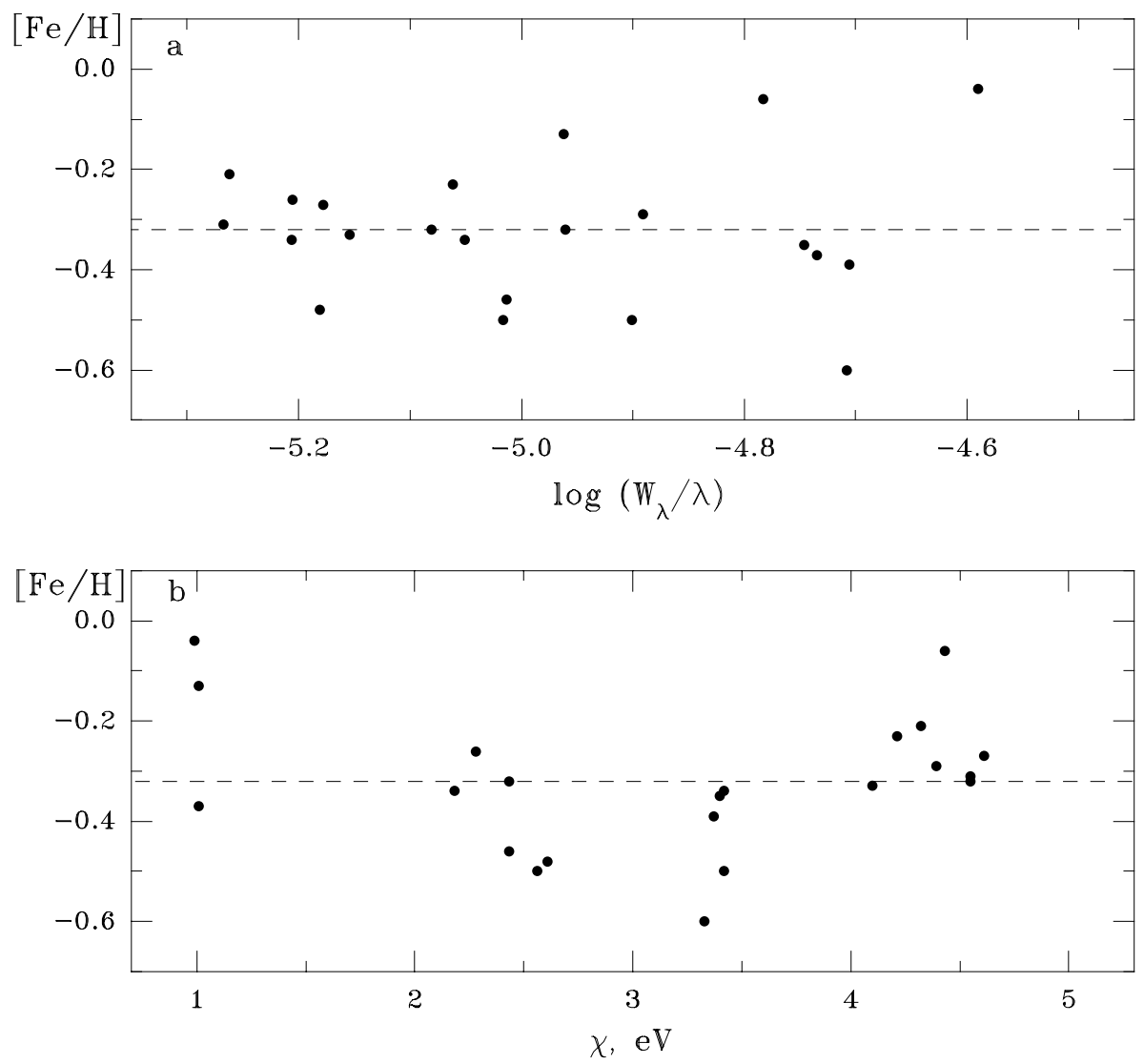

Fig. 5. Spectroscopic determination of the atmospheric parameters of HD 19993 using Fe I lines. Panel a) shows the iron abundances versus reduced equivalent widths. Panel b) shows the iron abundances versus lower exitation potentials.

Table 4. Average element abundances of HD 19993.

\begin{tabular}{rcrrrr}
\hline \hline Element & $\log \varepsilon(\mathrm{X})$ & {$[\mathrm{X} / \mathrm{H}]$} & {$[\mathrm{X} / \mathrm{Fe}]$} & $\sigma_{\text {tot }}$ & $n$ \\
\hline $\mathrm{C}$ I & 7.71 & -0.85 & -0.53 & 0.11 & 4 \\
O I & 8.33 & -0.50 & -0.18 & 0.09 & 3 \\
$\mathrm{Na} \mathrm{I}$ & 6.33 & 0.00 & +0.32 & 0.11 & 3 \\
$\mathrm{Mg}$ I & 7.17 & -0.41 & +0.09 & 0.11 & 2 \\
$\mathrm{Si}$ II & 7.03 & -0.52 & -0.20 & 0.09 & 1 \\
$\mathrm{Ca}$ I & 5.99 & -0.37 & -0.05 & 0.14 & 9 \\
$\mathrm{Sc}$ II & 2.67 & -0.43 & -0.11 & 0.17 & 5 \\
$\mathrm{Mn}$ I & 5.09 & -0.40 & -0.08 & 0.13 & 1 \\
Fe I & 7.20 & -0.32 & 0.00 & 0.14 & 22 \\
$\mathrm{Fe}$ II & 7.19 & -0.33 & -0.01 & 0.09 & 5 \\
$\mathrm{Ba}$ II & 1.99 & -0.14 & +0.18 & 0.19 & 2 \\
\hline
\end{tabular}

The absolute abundances (assuming $\log \varepsilon(\mathrm{H})=12.00$ ) are listed in Col. 2; the relative abundances with respect to hydrogen and iron are listed in Cols. 3 and 4, respectively; the abundance uncertainty is listed in Col. 5; the number of spectral lines used to derive the element abundance is listed in Col. 6 .

scattering (which include uncertainties in the measured $W_{\lambda}$ and $\log g f$ values): $\sigma_{\text {tot }}^{2}=\sigma^{2}{ }_{\Delta T_{\text {eff }}}+\sigma^{2}{ }_{\Delta \log g}+\sigma_{\Delta \xi_{\mathrm{t}}}^{2}+\sigma^{2}{ }_{\Delta W_{\lambda}+\Delta \log g f}$.

We carried out the abundance determination considering HD 19993 a single star, since it shows no visible signs of duplicity. The derived abundances are within the range of observed in single A-type giants (e.g., Erspamer \& North 2003).
According to Bessell et al. (1998), the intrinsic color-index $(B-V)_{0}$ of an atmosphere model with the parameters of HD 19993 is $0.15 \mathrm{mag}$, which gives a color-excess $E(B-V)=$ $0.35 \mathrm{mag}$. Applying the interstellar color-excess ratios from Bessell \& Brett (1988) to the other observed color-indices, one can see that HD 19993 exhibits no excess radiation between the $V$ and the $K$-band. The only deviation from the mean interstellar extinction law is seen in the $U-B$ color-index. The observed $E(U-B)=0.08 \mathrm{mag}$, which is $0.15 \mathrm{mag}$ lower than expected. This deviation may be due either to an anomalous extinction law in this direction or to the metal deficiency of the star.

Thus, HD 19993 is most likely an A7-A8 bright giant (luminosity type II) with a mild metal deficit (see Fig. 1a). The above results also allow us to estimate the object's luminosity and mass by comparing its observed parameters $\left(T_{\text {eff }}, \log g\right.$, and visual brightness) with theoretical pre- and post-main-sequence evolutionary tracks by Palla \& Stahler (1993) and Schaller et al. (1992), respectively (see Fig. 7). We found the best fit at $\log L / L_{\odot}=3.0 \pm 0.2$ and $M / M_{\odot}=4.8 \pm 0.8$ in the post-mainsequence domain, while the star is located above the birthline for the pre-main-sequence models. The derived luminosity corresponds to a distance $D=1.0 \pm 0.25 \mathrm{kpc}$ which is in agreement with the insignificant HIPPARCOS parallax.

We can also suggest an explanation for the observed $\mathrm{H} \alpha$ profile. It is unlikely to be formed in a spherical wind, since the profile would not have a central depression in the optically-thin case and would have a blue-shifted P Cyg type absorption in 

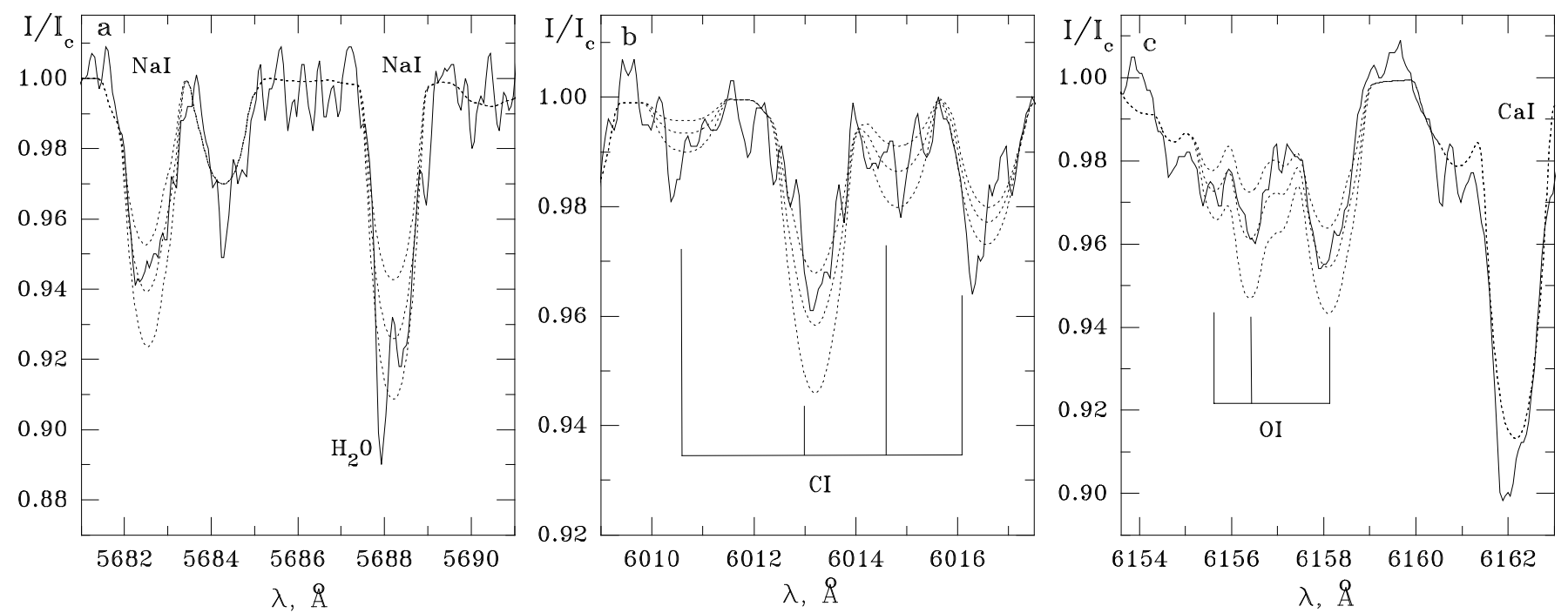

Fig. 6. Determination of the sodium, carbon and oxygen abundances of HD 19993. In all panels the observed spectrum is shown by solid lines and the synthetic spectra by dotted lines. The upper dotted line correspond to the lowest abundance, while the lower one corresponds to the highest abundance. Panel a) shows the wavelength region, containing the 5682.6 and $5688.2 \AA \mathrm{Na}$ I lines. The synthetic spectra were calculated for the sodium abundances of $[\mathrm{Na} / \mathrm{H}]=-0.20,0.00$, and +0.20 . The $5688.2 \AA$ line is contaminated with the $5687.5 \AA$ telluric water vapor line. Panel b) shows the wavelength region, containing the carbon lines. The synthetic spectra were calculated for the carbon abundances of $[\mathrm{C} / \mathrm{H}]=-1.00,-0.85$, and -0.70 . Panel c) shows the wavelength region, containing the oxygen lines. The synthetic spectra were calculated for the oxygen abundances of $[\mathrm{O} / \mathrm{H}]=-0.70,-0.50$, and -0.30 . The best fitting abundances are listed in Table 4 .

the optically-thick case. Stars with similar parameters to those of HD 19993 may have chromospheres, but they exhibit only a weak Ly- $\alpha$ emission due to the chromospheric gas (Marili et al. 1997). Double-peaked profiles are common in stars with gaseous CS disks (e.g., Be stars). The H $\alpha$ line of HD 19993 may be formed in such a disk, whose origin however is not clear. In Be stars the disk may be due either to a radiativelydriven wind (e.g., Bjorkman \& Cassinelli 1993) or to non-radial pulsations. The latter mechanism is more likely for a relatively cool star, such as HD 19993.

\section{2. $H D 29035$}

HD 29035 turned out to be a slightly reddened A0 V star $(E(B-V)=0.29 \mathrm{mag})$, in whose spectrum we detected only a few absorption lines. The line strengths indicate that it is a moderately rapidly rotating dwarf $\left(v \sin i \sim 150 \mathrm{~km} \mathrm{~s}^{-1}\right.$, see Fig. 3). The strong and narrow $\mathrm{Na} I \mathrm{D}$ lines as well as DIBs have an interstellar nature. The $\mathrm{H} \alpha$ line profile consists of a double-peaked component in emission (the peak separation is $\sim 200 \mathrm{~km} \mathrm{~s}^{-1}$ ), which is located inside the broad absorption wings. The latter are consistent with a luminosity type v, taking into account the uncertainties in the profile normalization mentioned above. No noticeable traces of the He I line $25876 \AA$ are seen in our spectra. This means that the line is intrinsically weak, putting an upper limit for the star's $T_{\text {eff }}$ at $\sim 11000 \mathrm{~K}$. The latter is consistent with the observed colour-indices and the $\mathrm{H} \alpha$ wings (Fig. 3a). The He I line might be partly filled-in with emission, but it seems unlikely given the weak $\mathrm{H} \alpha$ emission component.

HD 29035 was not included in the HIPPARCOS input catalog and had no parallax measurements. With a typical luminosity of an A0 V star $\left(M_{V}=0.8\right.$ mag, Straizhys $\&$ Kuriliene 1981, which corresponds to $\log L / L_{\odot}=1.7$ ), HD 29035 would be located at $D=250 \mathrm{pc}$. Optical photometry of nearby A-type stars by Tycho (Hog et al. 2000) suggest that, in this direction, $E(B-V) \sim 0.3 \mathrm{mag}$ is reached at $D \sim 350 \mathrm{pc}$. However since their spectral types were taken from HD (no luminosity types), we assumed that they are all dwarfs which makes the interstellar extinction law uncertain. On the other hand, HD 29035 is close to the Galactic plane where such a reddening at $0.3 \mathrm{kpc}$ is not unusual. Therefore, we conclude that HD 29035 has a luminosity close to that of a main-sequence star.

\section{Discussion}

The above results show that both stars have similar observed properties (e.g., a weak double-peaked $\mathrm{H} \alpha$ emission, almost no near-IR excess in combination with a noticeable far-IR excess), although their luminosities seem to be an order of magnitude different. Their spectral types turn out to be at different ends of the A-type range. These facts place some constraints on the objects' evolutionary state.

The $\mathrm{H} \alpha$ emission may be due to the star's youth (a premain-sequence Herbig Ae star). The strong IR excess of HD 29035 (see Table 1), detected in all 4 IRAS bands, and extended IRAS images support this suggestion. Iwata et al. (1999) pointed out a possible connection between IRAS 04331+5211 and HD 29035 and suggested that the star could be young, although no CO line emission was detected from it (Wouterloot \& Brand 1989). HD 29035 may be a classical Be star as was suggested for HD 4881, a B8 star with similar $\mathrm{H} \alpha$ emission and IR excess (Miroshnichenko et al. 1999). Classical Be stars are main-sequence objects with no CS dust, and the IR excess 


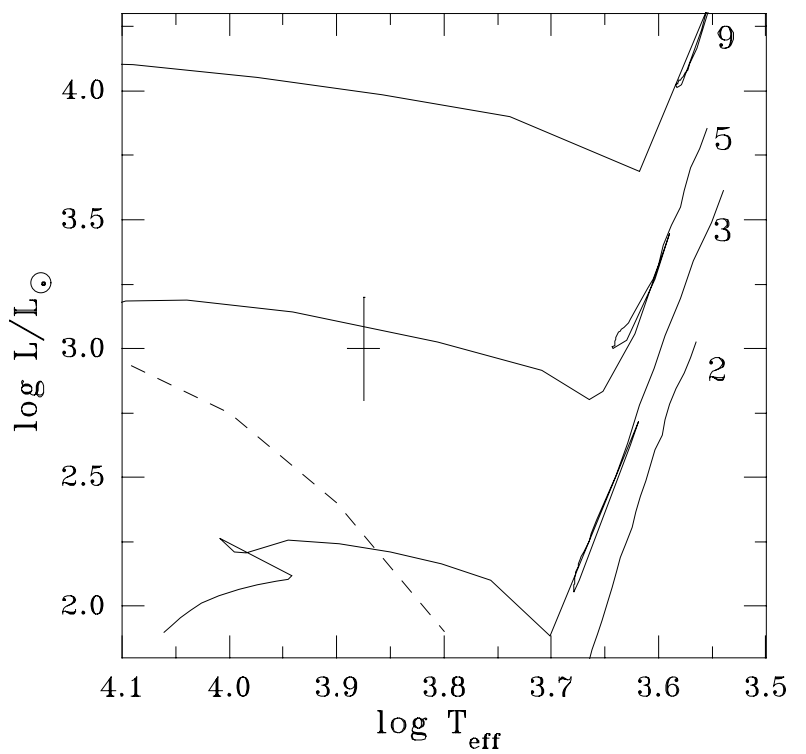

Fig. 7. The Hertzsprung-Russell diagram with a position of HD 19993. The evolutionary tracks from Schaller et al. (1992) are shown by solid lines. Numbers near the tracks indicate the corresponding mainsequence masses in solar units. The birthline for pre-main-sequence intermediate-mass stars by Palla \& Stahler (1993) is shown by a dashed line.

has to be explained by thermal radiation of nearby IS dust. Recently Kalas et al. (2002) showed that the $12 \mu \mathrm{m}$ excess of HD 4881 (if the IRAS measurement is confirmed by other observations) requires a CS dust contribution. Since it is even larger in HD 29035, its pre-main-sequence status seems to be more favorable.

HD 29035 does not belong to any known star formation region and shows no noticeable nebulosity on the Palomar Sky Survey image. The deep central depression in the $\mathrm{H} \alpha$ profile suggests a low inclination angle of the CS disk to the line of sight. Thus, HD 29035 is probably an isolated Herbig Ae star, a good candidate to search for the Algol-type photometric variations that are common in these close to edge-on systems (Grinin et al. 1991). At the same time, the SED in the near-IR region does not indicate the presence of excess radiation, which is usually noticeable in Herbig Ae stars. This implies the absence of hot dust near the star. On the other hand, the cool dust is definitely present. All of these properties are consistent with an interpretation that HD 29035 is a transition object between Herbig Ae/Be and Vega-type stars. In other words, it is a young star just before entering the main-sequence evolutionary stage. The IRAS photometry is consistent with the presence of dust with temperatures lower than $T_{\mathrm{d}} \sim 300 \mathrm{~K}$.

The location of HD 19993 well above both the mainsequence and the birthline for Herbig Ae/Be stars suggests that it is an evolved object. Therefore, the CS dust was most likely formed from the material ejected by the star during its earlier evolution. The absence of hot dust indicates that the dust formation process was not recent. Far-IR excesses due to CS dust, which is formed during the AGB evolutionary stage, are common in post-AGB stars. The luminosity of HD 19993 is consistent with that of a $1 M_{\odot}$ post-AGB object (e.g., Blöcker 1995).
However, its surface gravity is too large for a post-AGB object. Since dust formation around hot stars requires special conditions (e.g., high CS matter densities), the origin of the CS dust near HD 19993 is puzzling.

Another unusual issue is the $\mathrm{H} \alpha$ emission profile with a peak separation of $110 \mathrm{~km} \mathrm{~s}^{-1}$ and a full width at the photospheric wings level of $250 \mathrm{~km} \mathrm{~s}^{-1}$, which is observed in the spectrum of a star with $v \sin i=35 \mathrm{~km} \mathrm{~s}^{-1}$ and a critical rotation velocity of $225 \mathrm{~km} \mathrm{~s}^{-1}$. The well-separated emission peaks indicate that the gaseous disk orientation with respect to the line of sight is close to edge-on, and the full rotation velocity of the star is close to its observed projected value (if the star's and disk's rotation axes coincide with each other). It is hard to expect that such a slowly rotating star would develop a significant mass loss. This might have happened much earlier, when it was a main-sequence B-type star and might have rotated faster. Nevertheless, main-sequence B-type stars rarely exhibit both emission lines and dusty IR excesses.

Summarizing all the above information, we can draw the following conclusions:

1. Our spectroscopic observations of two bright stars with IRAS counterparts, HD 19993 and HD 29035, resulted in a discovery of the double-peaked emission $\mathrm{H} \alpha$ line profiles. The analysis of these data shows that HD 19993 is an A7/8 II type star, while HD 29035 is a B9/A0 v: star. The optical photometric and near-IR spectroscopic observations are in agreement with the above parameters.

2. The presence of the $\mathrm{H} \alpha$ emission and the IR excess suggests that HD 29035 is most likely a pre-main-sequence object and can be considered a Herbig Ae/Be star candidate. Its location outside of known star forming regions and the nearly edge-on disk-like envelope orientation make it similar to a group of isolated Herbig Ae stars.

3. The derived physical parameters of HD 19993 suggest that it is an intermediate-mass post-main-sequence star (if single). However, the nature of its CS dust seems to be puzzling.

Acknowledgements. A. S. Miroshnichenko and K. S. Bjorkman acknowledge support from NASA grant NAG5-8054. K. Bjorkman is a Cottrell Scholar of the Research Corporation, and gratefully acknowledges their support. Drake acknowledges a fellowship of Observatório Nacional/MCT (Brazil) and computing support from FAPERJ (Brazil) under grant E-26/171.647/99. Rudy, Lynch, Mazuk, and Venturini were supported by The Aerospace Corporation's Independent Research and Development program. Miroshnichenko and Kusakin thank K. S. Kuratov for his help in organizing the photometric observations at the Tien-Shan Observatory. This research has made use of the SIMBAD database operated at CDS, Strasbourg, France, and the VALD database operated at the Institute für Astronomie, Wien, Austria.

\section{References}

Alonso, A., Arribas, S., \& Martinez Roger, C. 1996, A\&AS, 117, 227

Anders, E., \& Grevesse, N. 1989, Geoch. Cosmoch. Acta, 53, 197

Barklem, P. S., Stempels, H. C., Allende Prieto, C., et al. 2002, A\&A, 385,951

Bessell, M. S., \& Brett, J. M. 1988, PASP, 100, 1134 
Bessell, M. S., Castelli, F., \& Plez, B. 1998, A\&A, 333, 231

Bjorkman, J. E., \& Cassinelli, J. P. 1993, ApJ, 409, 429

Blöcker, T. 1995, A\&A, 299, 755

Coté, J., \& van Kerkwijk, M. H. 1993, A\&A, 274, 870

Denisenkov, P. A., \& Ivanov, V. V. 1987, Soviet Astr. Lett., 13, 214

Erspamer, D., \& North, P. 2003, A\&A, 398, 1121

ESA 1997, The Hipparcos and Tycho Catalogues ESA SP-1200

Grinin, V. P., Kiselev, N. N., Chernova, G. P., Minikulov, N. Kh., \& Voshchinnikov, N. V. 1991, Ap\&SS, 186, 233

Hoffleit, D., \& Warren Jr., J. H. 1991, The Bright Star Catalog, 5th Revised Ed., Astronomical Data Center, NSSDC/ADS

Hog, E., Fabricius, C., Makarov, V. V., et al. 2000, A\&A, 355, L27

Iwata, I., Okumura, S., \& Saito, M. 1999, PASJ, 51, 653

Kalas, P., Graham, J. R., Beckwith, S. V. W., Jewitt, D. C., \& Lloyd, J. P. 2002, ApJ, 567, 999

Khaliullin, Kh., Mironov, A. V., \& Moshkaliov, V. G. 1985, Ap\&SS, 111,291

Kornilov, V. G., Volkov, I. M., Zakharov, A. I., et al. 1991, Proc. of the Sternberg Astron. Inst., v.63

Kupka, F., Piskunov, N. E., Ryabchikova, T. A., Stempels, H. S., \& Weiss, W.W. 1999, A\&AS, 138, 119

Kurucz, R. L. 1993, CD-ROM No. 13, ATLAs9 Stellar Atmosphere Programs and $2 \mathrm{~km} / \mathrm{s}$ Grid (Cambridge: Smithsonian Astrophysical Obs.)

Kurucz, R. L. 1994, Smithsonian Astrophys. Obs., CD-ROM No. 19

Lambert., D. L. 1992, in Instabilities in evolved super- and hypergiants, ed. H. de Jager, Nieuwenhuijzen (North-Holland, Amsterdam), 156

Lambert., D. L., Heath, J. E., Lemke, M., \& Drake, J. 1996, ApJS, 103, 183

Mannings, V., \& Barlow, M. J. 1998, ApJ, 497, 330

Marili, E., Catalano, S., Freire Ferrero, R., et al. 1997, A\&A, 317, 521

McCarthy, J. K., Sandiford, B. A., Boyd, D., \& Booth, J. 1993, PASP, 105,881

Merrill, P. W., \& Burwell, C. G. 1933, ApJ, 78, 87
Miroshnichenko, A. S., Mulliss, C. L., Bjorkman, K. S., et al. 1999, MNRAS, 302, 612

Miroshnichenko, A. S., Chentsov, E. L., Klochkova, V. G., et al. 2000, A\&AS, 147, 5

Miroshnichenko, A. S., Bjorkman, K. S., Chentsov, E. L., et al. 2001, A\&A, 377, 854

Oudmaijer, R. D., van der Ween, W. E. C. J., Waters, L. B. F. M., et al. 1992, A\&AS, 96, 625

Palla, F., \& Stahler, S. 1993, ApJ, 418, 414

Piskunov, N. E., Kupka, F., Ryabchikova, T. A., Weiss, W. W., \& Jeffery, C. S. 1995, A\&AS, 112, 525

Reed, B. C. 1998, ApJS, 115, 271

Rosendhal, J. D. 1974, ApJ, 187, 261

Rozenzweig, P., \& Anderson, L. 1993, ApJ, 411, 207

Rudy, R. J., Puetter, R. C., \& Mazuk, S. 1999, AJ, 118, 666

Savage, B. D., \& Mathis, J. S. 1979, ARA\&A, 17, 73

Schaller, G., Schaerer, D., Meynet, G., \& Maeder, A. 1992, A\&AS, 96, 269

Skrutskie, M. F., Schneider, S. E., Stiening, R., et al. 1997, in Proc. Workshop The Impact of Large Scale Near-IR Sky Surveys, 25

Sneden, C. 1973, ApJ, 184, 839

Sneden, C., Kraft, R. P., Prosser, C. F., \& Langer, G. E. 1991, AJ, 102, 2001

Straizhys, V., \& Kuriliene, G. 1981, Ap\&SS, 81, 353

Takeda, Y., \& Takada-Hidai, M. 1994, PASJ, 46, 395

Uesugi, A., \& Fukuda, I. 1970, Catalogue of rotational velocities of the stars, Univ. of Kyoto

Venn, K. A. 1995, ApJSS, 99, 659

Verdugo, E., Talavera, A., \& Gómez de Castro A. I. 1999, A\&A, 346, 819

Waters, L. B. F. M., Coté, J., \& Lamers, H. J. G. L. M. 1987, A\&AS, 172, 225

Weaver, Wm. B., \& Jones, G. 1992, ApJS, 78, 239

Wouterloot, J. G. A., \& Brand, J. 1989, A\&AS, 80, 149 